East African Medical Journal Vol. 86 No. 1 January 2009

HYDROTUBATION IN THE MANAGEMENT OF FEMALE INFERTILITY: OUTCOME IN LOW RESOURCE SETTINGS

A. G. Adesiyun, FWACS, Department of Obstetrics and Gynaecology, Ahmadu Bello University Teaching Hospital, Shika - Zaria Kaduna State, Nigeria, B. Cole, MBBS, Echo Scan Services Limited, 4, Katsina Road - Kaduna, Kaduna State, Nigeria and P. Ogwuche, MBBS, Alba Clinic and Medical Center, 25, Constitution Road - Kaduna, Kaduna State, Nigeria

Request for reprints to: Dr. A. G. Adesiyun, P. O.Box 204, Kaduna - Kaduna State, Nigeria

\title{
HYDROTUBATION IN THE MANAGEMENT OF FEMALE INFERTILITY: OUTCOME IN LOW RESOURCE SETTINGS
}

\author{
A. G. ADESIYUN, B. COLE and P. OGWUCHE
}

\begin{abstract}
Objectives: To determine the outcome of therapeutic hydrotubation (tubal flushing) in patients with tubal infertility and unexplained infertility.

Design: Prospective non-randomised observational study.

Setting: Alba hospital, a five bed multidisciplinary private hospital in Kaduna city, Nigeria.

Subjects: Infertile women with tubal and unexplained infertility.

Results: Two hundred and fifty seven patients that had therapeutic hydrotubation over 7.5 years were analysed. The age range was 21 to 44 years with mean of 34.5 years. Secondary infertility was found in $209(81.3 \%)$ patients and primary infertility in 166 (64.6\%) patients. Of the 257 patients, $134(52.1 \%)$ had had induced abortion. Indications for hydrotubation were bilateral perifimbrial adhesion (incomplete tubal occlusion) $47.9 \%$, bilateral fimbrial end occlusion $24.9 \%$, bilateral cornual blockage $16 \%$ and unexplained infertility in $\mathbf{1 1 . 3 \%}$ of cases. One hundred and nine conceptions were recorded in this study and the overall conception rate was $42.4 \%$. Likewise, percentage ratio of conception in these 154 patients with evidence of post - hydrotubation tubal patency was $70.8 \%$. Pregnancy outcome of the 109 conceptions are term pregnancy $84.4 \%$, preterm pregnancy $9.2 \%$, miscarriage $4.6 \%$ and ectopic pregnancy in $1.8 \%$ of the conceptions. Recorded complications were pelvic pain in $177(68.9 \%)$ patients and vaginal bleeding in $63(24.5 \%)$ patients.

Conclusion: With good case selection, therapeutic hydrotubation may be beneficial in resource poor countries, especially in patients with incomplete tubal occlusion (bilateral perifimbrial adhesions) and as part of treatment for unexplained infertility.
\end{abstract}

\section{INTRODUCTION}

Infertility remains a major reason for gynaecological consultation in developing countries. It is associated with social consequences like marital instability, divorce, polygamy, prostitution and suicide in extreme cases. In the tropics, tubal factors remain a common cause of female infertility (1). This is due to high prevalence of sexually transmitted diseases in our setting. Optimal diagnosis and treatment of tubal factor, though standardised, vary from place to place, depending on how developed the health care services are. Methods of managing tubal factors in infertility range from gaseous insufflation, hydrotubation, microsurgery, hysteroscopic catheterisation and in vitro fertilisation (2).

Microsurgical operations and hysteroscopic catheterisation technology are not available in our setting. Although there are very few in vitro fertilisation (IVF) units, the services are usually unaffordable and out of reach to majority of patients that may benefit from it. The recently introduced National Health Insurance Scheme in the country does not cater for this service. It costs between four to six thousand United States Dollars for one IVF cycle. Against this background, we performed hydrotubation on most of these patients as a last resort. 


\section{MATERIALS AND METHODS}

This was a prospective study carried out in a privately owned 50 bed multidisciplinary hospital in Kaduna City, Nigeria. The study period was from January 1999 through to June 2006. During this period, 291 hydrotubation (HTB) procedures were done, but 257 cases that were followed up for at least six months post-procedure were analysed in this study.

The inclusion criteria and indications for hydrotubation were bilateral tubal pathology and unexplained infertility. Patients with hydrosalpinx, occlusion in the mid portion of the fallopian tubes and unilateral patent tubes were excluded from this study. The patients were screened and appropriately treated for pelvic infection before they were subjected to hysterosalpingogram (HSG) and subsequently hydrotubation. Patients also had pelvic sonogram pre and post procedure. The essence of the pre-HTB pelvic sonogram was to rule out free fluid collection in the pelvis, so as not to mistake it for successful HTB evidenced by free fluid collection in the pelvis post-HTB. Laparoscopy and dye hydrotubation was not done for most patients, due to affordability and availability to laparoscopic evaluation in low resource setting like ours.

Hydrotubation was carried out as an outpatient procedure during the $6^{\text {th }}$ to the $10^{\text {th }}$ day of a menstrual cycle. The patients received short acting intravenous (sodium thiopentone) anaesthetic agent. The cervix was canullated with a Leech-Wilkinsons canullae and $50 \mathrm{mls}$ loaded syringe attached to the canullae was used to flush the fallopian tubes. The solution for HTB was made up of normal saline, crystalline penicillin and hydrocostisone; 50 to $100 \mathrm{mls}$ of the solution was used per procedure.

Post procedure, patients were transferred to the recovery room, where they were given a dose of injectable non-steroidal anti-inflammatory analgesia. Following full recovery, they were discharged on analgesics and prophylactic antibiotics. Repeat HTB was done for some patients in subsequent menstrual cycle(s) due to marked resistance and reflux of the solution.

They were followed up in the gynaecological clinic. Their first visit was the next menstrual cycle, during which ultrasound scan was performed to confirm ovulatory cycle and were counselled on timed intercourse. Those patients with anovulatory cycle were placed on clomiphene citrate in subsequent menstral cycles.

The 257 patients that had hydrotubation were divided into four main groups as follows:

Group A: Patients that had bilateral cornual occlusion of the fallopian tubes on HSG.

Group B: Patients that had bilateral fimbrial end tubal occlusion on HSG.
Group C: Patients that had bilateral peri-fimbrial adhesion evidenced by loculated and restricted spillage of contrast on HSG.

Group D: Patients with unexplained infertility and evidence of patent tubes on HSG.

\section{RESULTS}

A total of 257 patients that had hydrotubation over a period of 7.5 years were analysed. Of the 257 patients, $154(59.5 \%)$ had evidence of post - HTB patency of the fallopian tube(s) confirmed with sonogram and $109(42.4 \%)$ patients out of the latter went ahead to conceive.

Patients profile: The mean age of the patients was 34.5 years with age range of 21 to 44 years. Of the 357 patients, $99(38.5 \%)$ had never delivered before and the highest number of childbirths amongst the category of patients that had delivered before was three. Seventy five $(29.2 \%)$ patients had no history of previous abortions. Spontaneous abortion accounted for $48(18.7 \%)$ patients of cases and induced abortion 118 (45.9\%)patients while the remaining $\operatorname{six}(6.2 \%)$ patients had both spontaneous and induced abortion. When extrapolated, 134 $(52.1 \%)$ (Table 1) patients had previous history of induced abortion.

Secondary infertility was the commonest type of infertility in $209(81.3 \%)$ patients and $166(64.6 \%)$ patients had been infertile for durations of 1 to 5 years. Frequency of hydrotubation in this study showed that $233(90.7 \%)$ patients had it once, 21 $(8.2 \%)$ patients twice and three $(1.2 \%)$ patients had it thrice.

Indications for hydrotubation and pregnancy attainment: The indications for hydrotubation showed that patients with bilateral perifimbrial adhesions, that is incomplete tubal occlusion (group C) accounted for $123(47.9 \%)$ of cases. This was followed by bilateral fimbrial end blockage (group B) 64 (24.9\%) patients, bilateral cornualblockage (group A) 41 (16\%) patients and patients with unexplained infertility (group D) accounted for $29(11.3 \%)$ patients of cases.

Percentage ratio of conception per indications for hydrotubation, showed that the highest conception per indication $(56.1 \%)$ was achieved in group C and the least $(24.3 \%)$ in group $\mathrm{A}$. This reveals that hydrotubation is more effective in treating patients with incomplete tubal occlusion (perifimbrial adhesions), followed by unexplained infertility, perifimbrial occlusion and cornual blockage (Table 2). In this study, the overall conception rate following hydrotubation was $42.4 \%$. 


\section{Table 1}

Profile of patients $(n=257)$

\begin{tabular}{lcc}
\hline Variable & No. & $(\%)$ \\
\hline Age (years) & 89 & 34.6 \\
$20-30$ & 134 & 52.2 \\
$31-40$ & 34 & 13.2 \\
$41-50$ & & \\
Previous delivery & 99 & 38.5 \\
0 & 113 & 44.0 \\
1 & 28 & 10.9 \\
2 & 17 & 6.6 \\
3 & & \\
Previous abortion & 75 & 29.2 \\
Nil abortion & 48 & 18.7 \\
Spontaneous & 118 & 45.9 \\
Induced & 16 & 6.2 \\
Spontaneous and induced & & \\
Type of infertility & 48 & 18.7 \\
Primary & 209 & 81.3 \\
Secondary & & \\
Duration of infertility (years) & 166 & 28.0 \\
$1-5$ & 72 & 64.6 \\
6 - 10 & 19 & 7.4 \\
$>11$ & & \\
Frequency of hydrotubation & 233 & 1.2 \\
Once & 21 & \\
Twice & 3 & \\
Thrice & & \\
\hline
\end{tabular}

Table 2

Group distribution of patients and post hydrotubation attainment of conception $(n=257)$

\begin{tabular}{cccccc}
\hline & & Group & \multicolumn{2}{c}{$\begin{array}{c}\text { Attainment of } \\
\text { conception }\end{array}$} & $\begin{array}{c}\text { Percentage ratio of } \\
\text { conception per group } \\
(\%)\end{array}$ \\
\hline No. & $(\%)$ & No. & $(\%)$ & 24.3 \\
B & 41 & 16.0 & 10 & 9.2 & 29.7 \\
C & 64 & 24.9 & 19 & 17.4 & 36.1 \\
D & 123 & 47.9 & 69 & 63.3 & 38.0 \\
\hline
\end{tabular}

Overall rate of conception $=42.4 \%$ 
Post hydrotubation tubal patency and attainment of conception: Tubal patency was confirmed in 154 patient post - HTB with pelvic sonography and 109 conceptions were subsequently recorded. All patients that conceived in this study had evidence of tubal patency post - HTB. This gives us an overall percentage ratio of $70.8 \%$ conceptions following post - HTB evidence of tubal patency.

Except for patients with unexplained infertility (group D), the percentage ratio of conception positively correlated with evidence of tubal patency following hydrotubation (Table 3). This means that patients with post HTB confirmation of tubal patency with sonogram have more chances to conceive compared to patients with no evidence of post -HTB tubal patency.

Post hydrotubation attainment of conception with or without clomiphene citrate: From Table 4, a significant percentage $(63.6 \%)$ of patients with unexplained infertility (group D) had to use clomiphene citrate before they could conceive post hydrotubation. On the contrary, greater percentage of patients in group A $(80 \%)$, group B $(68.4 \%)$ and group C $(79.7 \%)$ did not use clomiphene citrate before they achieved conception post hydrotubation.

Complications of hydrotubation and pregnancy outcome: There was no major complication recorded in this study that warrants hospitalisation. Minor complication/complaints encountered were pelvic pain in $177(68.9 \%)$ patients and vaginal bleeding in $63(24.5 \%)$ patients. These patients were treated on outpatient basis.

The outcome of the 109 conceptions revealed that $92(84.4 \%)$ ended up in term pregnancy, 10 $(9.2 \%)$ in preterm delivery and five $(4.6 \%)$ in miscarriage. Two $(1.8 \%)$ conceptions were ectopic pregnancies (Table 5).

Table 3

Evidence of post hydrotubation tubal patency and conception rate in the groups

\begin{tabular}{|c|c|c|c|c|c|}
\hline \multirow[t]{2}{*}{ Group } & \multicolumn{2}{|c|}{$\begin{array}{l}\text { Post }- \text { HTВ } \\
\text { tubal patency }(n=154)\end{array}$} & \multicolumn{2}{|c|}{$\begin{array}{c}\text { Attainment of } \\
\text { conception }(\mathrm{n}=109)\end{array}$} & \multirow{2}{*}{$\begin{array}{c}\text { Percentage ratio } \\
\text { of conception } \\
\text { post-HTB patency } \\
(\%)\end{array}$} \\
\hline & No. & $(\%)$ & No. & $(\%)$ & \\
\hline A & 17 & 11.0 & 10 & 9.2 & 58.8 \\
\hline B & 26 & 16.9 & 19 & 17.2 & 73.1 \\
\hline $\mathrm{C}$ & 82 & 53.3 & 69 & 63.3 & 84.1 \\
\hline $\mathrm{D}$ & 29 & 18.8 & 11 & 10.1 & 37.9 \\
\hline
\end{tabular}

Overall percentage ratio of conception following evidence of post - HTB tubal patency $=70.8 \%$

${ }^{*} \mathrm{HTB}=$ Hydrotubation

Table 4

Group distribution of patients and post-HTB conception with or without clomiphene citrate

\begin{tabular}{lccccc}
\hline Group & $\begin{array}{c}\text { Number of } \\
\text { conception }\end{array}$ & Clomiphene & Conception with & \multicolumn{2}{c}{$\begin{array}{c}\text { Conception without } \\
\text { Citrate }\end{array}$} \\
\hline A & 10 & 8 & $80.0 \%$ & 2 & $20.0 \%$ \\
B & 19 & 13 & $68.4 \%$ & 6 & $31.6 \%$ \\
C & 69 & 55 & $79.7 \%$ & 14 & $20.3 \%$ \\
D & 11 & 4 & $36.4 \%$ & 7 & $63.6 \%$ \\
\hline
\end{tabular}

\footnotetext{
* $\mathrm{HTB}=$ Hydrotubation
} 
Table 5

Outcome of pregnancy $(\mathrm{n}=109)$

\begin{tabular}{lcc}
\hline Variable & No. & $(\%)$ \\
\hline Term pregnancy & 92 & 84.4 \\
Preterm pregnancy & 10 & 9.2 \\
Miscarriage & 5 & 4.6 \\
Eccyesis & 2 & 1.8 \\
\hline
\end{tabular}

\section{DISCUSSION}

The assessment of fallopian tubes patency is an integral and pertinent step in the evaluation of female infertility (3). Also, the treatment of tubal factor in infertility management is most difficult and subject to debates (2). In this study, hysterosalpingogram was used to assess patency of the fallopian tubes though not as accurate as laparoscopy and dye test, it has been reported to have positive predictive value between 61.7 to $73 \%(4,5)$.

Hydrotubation for therapeutic intervention was employed as a last resort of treatment for these patients because it is a simple, cheap and minimally invasive procedure with low risk of complications. The inclusion of antibiotic (crystalline penicillin) and steroid (hydrocortisone) in the formulation of hydrotubation solution used in this study, was based on study by Johnson and Watson (6) that reported reduced infection and increased pregnancy with live birth rate when used after reproductive pelvic surgery to enhance fertility.

Other studies $(7,8)$ have reported fertility enhancing effect of tubal flushing especially with oil soluble medium, we used saline solution in our study due to the side effects of oil media and because our intervention was principally for therapeutic reason. Oil media have been associated with persistent contrast medium within the pelvis, allergy or anaphylaxis reaction from intravasation and formation of lipogranuloma on a long term. It is therefore not surprising that we only recorded minor complaints with the use of water medium in our study. The patients in our series had pre - HTB screening for pelvic infection and post-HTB antibiotic prophylaxis. We were guided in this direction by the recommendation of the Royal College of Obstetricians and Gynaecologists (9) for women going for uterine instrumentation and the finding of clinical infection following hysterosalpingogram by Forsey et al (10).

Secondary infertility was predominant in our study. Similarly, Bello (11) found tubal pathology to be significantly associated with secondary infertility than primary infertility. Also noteworthy from our series, is that more than half of our patients had had induced abortion, most likely in an unsafe environment due to illegality attached to it in our country. Therefore, the contribution of unsafe abortion in the aetiopathogenesis of tubal disease in our infertile women cannot be overestimated.

We employed post - HTB sonogram to assess patency of the fallopian tube(s) following the procedure. This was based on results of other studies that reported accuracy rate of diagnostic saline HTB ranging from 82.9 to $87.1 \%(5,12)$. Excluding patients with unexplained infertility, our results show that the chances of conception increased with evidence of post - HTB tubal patency. Furthermore, evidence of post - HTB tubal patency is of most predictive value in terms of conception rate in patients with perifimbrial adhesions and least in patients with unexplained infertility. On the whole, the $70.8 \%$ conception rate recorded in patients with post - HTB evidence of tubal patency, also help to buttress the effectiveness of hydrotubation in the evaluation of tubal patency.

In this study, the overall conception rate of $42.4 \%$ recorded in 257 patients that had therapeutic hydrotubation is encouraging, when we considered the "hopeless" nature of these cases in a resource constrained setting like ours. We also found this procedure to be more effective in patients with perifimbrial adhesions (incomplete tubal occlusion), followed by patients with unexplained infertility when combined with clomiphene citrate for ovulation induction. The hypothesis by Sulak et al (13) that proximal tubal blockage is caused by plugs of amorphous materials that occlude the tubal lumen without the presence of any tubal wall damage, made us to include bilateral cornual end tubal blockage as one of the indiccution for hydrotubation. The pregnancy outcome is also encouraging, with term pregnancies mainly recorded. Outcomeof pregnancies are comparable to figures reported with the use of oil-soluble media (14). Though use of oil-soluble contrast is associated with an increase in the odds if live births but not pregnancy rates when compared with water soluble media (14).

The reliability on HSG for assessing the fallopian tubes was a major limitation of this study. Nonetheless, we found therapeutic HTB beneficial in the management of female infertility, especially incomplete tubal occlusion and unexplained infertility in resource poor settings, where modern technology for management are mostly unavailable and expensive. However, a randomised controlled trial with pre - HTB laparoscopic evaluation of the fallopian tubes is recommended, more especially for cases with incomplete tubal occlusion and unexplained infertility. 


\section{REFERENCES}

1. Cates, W., Farley, T.M.M. and Rowe, P.J. Worldwide pattern of infertility. Is Africa different? Lancet. 1985; 14: 596 - 598 .

2. El-Mowafi, D.M. and Ngoh, N.N. Management of tubal obstructions. Surg. Technol. Int. 2005; 14: 199 $-212$.

3. Lupascu, I., Veghes, S., Solomitchi, V., et al. Sonohysterosalpingography in the assessment of tubal infertility. Rev. Med. Chir. Soc. Med. Nat. Iasi. 2003; 107: $841-845$.

4. Vasiljevic, M., Ganovic, R., Jovanovic, R. and Markovic, A. Diagnostic value of hysterosalpingography and laparoscopy in infertile women. Srp. Arh. Celok. Lek. 1996; 124: 135 - 138.

5. Zuo, W. and Wang, P. Comparative study on assessment of tubal patency among tubal insufflation, hydrotubation, hysterosalpingogram and chromotubation under laparoscopy. Zhonghua Fu Chan Ke Za Zhi. 1996; 31: 29 - 31.

6. Johnson, N.P and Watson, A. Cochrane review: postoperative procedure for improving fertility following pelvic reproductive surgery. Hum. Reprod. Update. 2000; 6: 259 - 267.

7. Johnson, N.P., Farguhar, C.M., Hadden, W.E., et al. The flush trial - flushing with lipiodal for unexplained (and endometriosis - related) subfertility by hysterosalpingography: a randomized trial. Hum. Reprod. 2004; 19: 2043 - 2051.

8. Vandekerckhove, P., Watson, A., Lilford, R., et al. Oil soluble versus water soluble media for assessing tubal patency with hysterosalpingography or laparoscopy in subfertile women. Cochrane Database Syst. Rev. 2000; (2): CD 000092.

9. RoyalCollege of Obstetricians and Gynecologists. The initial investigation and management of the infertile couple. RCOG Press, London, UK. 1996, Pg 12.

10. Forsey, J.P., Caul, E.O., Paul, I.D. and Hull, M.G.R. Chlamydia trachomatis, tubal disease and the incidence of symptomatic and asymptomaticinfection following hysterosalpingography. Hum. Reprod. 1990; 5: $444-447$.

11. Bello, T.O. Tubal abnormalities on hysterosalpingography in primary and secondary infertility. West. Afr. J. Med. 2006; 25: 130 - 133.

12. Kalogirou, D., Antoniou, G., Botisis, D., et al. Is color Doppler necessary in the evaluation of tubal patency by hysterosalpongo-contrast sonography. Clin. Exp. Obstet. Gynecol. 1997; 24: 101 - 103.

13. Sulak, P.J., Letterie, G.S., Coddington, C.C., et al. Histology of proximal tubal occlusion. Fertil. Steril. 1987; 48: 437 - 440.

14. Johnson, N., Vandekerckhove, P., Watson, A., et al. Tubal flushing for subfertility. Cochrane Database Syst. Rev. 2002; (3): CD003718. 